\title{
Manejo endovascular de la hemorragia digestiva, experiencia del Hospital Universitario Clínica San Rafael
}

\section{Endovascular management of gastrointestinal bleeding, experience at Hospital Universitario Clínica San Rafael}

\author{
César Eduardo Jiménez ${ }^{1}$ Leonardo Randial², Fernando Quiroga ${ }^{3}$
}

\author{
Jefe, Servicio de Cirugía Vascular, Hospital Universitario Clínica San Rafael; director científico, Centro Integral de Heridas y \\ Enfermedades Vasculares, CINTHEV, Bogotá, D.C., Colombia \\ 2 Cirujano vascular, Hospital Universitario Clínica San Rafael, Bogotá, D.C., Colombia \\ 3 Epidemiólogo clínico, Fundación Sinergia y Sociedad, Bogotá, D.C., Colombia
}

\begin{abstract}
Resumen
Introducción. La hemorragia digestiva ocasiona el $2 \%$ de las hospitalizaciones; se clasifica en alta o baja, y la primera se presenta en el $80 \%$ de casos. Después de la estabilización hemodinámica, se determinan la causa y el tratamiento mediante la endoscopia; no obstante, esta falla en Io a $20 \%$ de los casos, y del 15 al $20 \%$ requieren cirugía mayor, con una mortalidad de más del $40 \%$. El tratamiento para la hemorragia digestiva mediante la formación de émbolos de los vasos mesentéricos, es una conducta bien establecida, produce buenos resultados, evita la cirugía y disminuye la morbimortalidad.

Objetivos. Determinar la indicación y el éxito del tratamiento endovascular para la hemorragia digestiva en nuestra institución.

Materiales y métodos. Estudio retrospectivo y descriptivo, se incluyeron to pacientes que requirieron la urgente formación de émbolos por falla o imposibilidad del manejo endoscópico, y que presentaban gran riesgo quirúrgico y anestésico con la técnica abierta.

Se evaluaron la causa de la hemorragia, la arteria comprometida, los hallazgos angiográficos, la hemoglobina antes y después de la formación de los émbolos, la reincidencia de la hemorragia, las complicaciones, la necesidad de intervención quirúrgica, la eficacia del procedimiento y la mortalidad a 30 días.

Resultados. Todos los pacientes se intervinieron por vía endovascular, para la oclusión selectiva de las arterias comprometidas. La hemorragia se controló en todos ellos. Se presentaron dos muertes tempranas (<30 días) no asociadas con el procedimiento. No hubo complicaciones secundarias a la formación de los émbolos o al acceso percutáneo y, tampoco, necesidad de cirugías mayores posteriores para controlar la hemorragia.
\end{abstract}

Fecha de recibido: 7/10/2018 - Fecha de aceptación: 4/12/2018

Correspondencia: César Eduardo Jiménez, MD, Avenida Boyacá Nº4-42, Bogotá, D.C., Colombia, Teléfono: (571) $492-9949$.

Correo electrónico: cesarejmd@yahoo.com

Citar como: Jiménez CE, Randial L, Quiroga F. Manejo endovascular de la hemorragia digestiva, experiencia del Hospital Universitario Clínica San Rafael. Rev Colomb Cir. 2019;34:234-44. https://doi.org/10.30944/20117582.436

Este es un artículo de acceso abierto bajo una Licencia Creative Commons - BY-NC-ND https://creativecommons.org/licenses/by-nc-nd/4.0/deed.es 
Conclusión. Los métodos endovasculares para controlar la hemorragia digestiva son eficaces, no se acompañan de complicaciones, y disminuyen la morbimortalidad y la necesidad de cirugías mayores. Se requieren estudios con mayor número de pacientes para lograr un mayor grado de certeza.

Palabras clave: hemorragia gastrointestinal; enfermedades vasculares; oclusión vascular; procedimientos endovasculares; dispositivos de cierre vascular; endoscopía del sistema digestivo.

\begin{abstract}
Introduction: Gastrointestinal bleeding is a common problem, which represents $2 \%$ of hospitalizations. It is classified as high or low depending on their origin; $80 \%$ of cases are high bleeders. After hemodynamic stabilization, endoscopy is very important to determine the cause and carry out treatment, which in some cases is unsuccessful or cannot be performed, and $15 \%-20 \%$ will require major surgery with a mortality rate of over $40 \%$. Occlusion of mesenteric vessels, as a treatment for gastrointestinal bleeding is a well defined and successful therapeutic, avoiding major surgery and reducing morbidity and mortality, it is a procedure with very low incidence of complications and repeated bleeding. We studied: the cause of bleeding, the compromised artery, the angiographic findings, the pre and post embolization hemoglobin, re-bleeding complications, need for additional surgery after embolization effectiveness of the procedure to control bleeding, and mortality at 30 days. Objectives: To determine the indication and success of endovascular treatment for the management of gastrointestinal bleeding in our institution
\end{abstract}

Materials and methods: A retrospective and descriptive study.

Results: Ten patients ( 5 females, 5 males) are included, with an average age of 59.8 years; in six cases the bleeding was due to proximal acid-peptic disease, two patients with diverticular disease performed, and two patients bleeding due to gastroduodenal neoplastic disease; all patients required urgent embolization in view of failure or inability to endoscopic management, associated with progressive anemia, active bleeding, schock and requirement of more than 3 packed red blood cells; associated with high surgical and anesthetic risk for open surgery. All patients underwent endovascular procedure with selective embolization and the bleeding stop with hemodynamic stabilization, two early deaths (<3odays) were presented but not associated with the procedure; there were no complications secondary to embolization or percutaneous access, nor was need for further surgery after the procedure to control bleeding

Conclusion: In our experience the use of endovascular methods to control gastrointestinal bleeding is effective and uncomplicated; reducing morbidity and mortality and the need for major surgery. More studies are needed to determine the number of patients a higher level of evidence

Key words: gastrointestinal hemorrhage; vascular diseases; vascular occlusion; endovascular procedures; vascular closure devices; endoscopy, digestive system.

\section{Introducción}

La hemorragia digestiva ocasiona el $2 \%$ de las hospitalizaciones. Se denomina alta (80 \%) cuando su origen se localiza por encima del ligamento de Treitz, o baja (20 \%), cuando lo hace por debajo del mismo '. Después de la estabilización hemodinámica del paciente, se determinan la causa y el tratamiento mediante la endoscopia; no obstante, esta falla en Io a $20 \%$ de los casos, y del I5 al $20 \%$ de los pacientes requieren ciru- gía mayor, con una mortalidad de más del $40 \%$. Por esto, se considera que los procedimientos mínimamente invasivos son una buena opción terapéutica ${ }^{\mathrm{I}-3}$.

El diagnóstico y el tratamiento de la hemorragia gastrointestinal son un desafío para los diferentes especialistas (cirujanos generales, gastroenterólogos, internistas) que intervienen en el manejo de estos pacientes. La hemorragia digestiva alta tiene una incidencia anual de I4 a 
50 casos por IO0.00o habitantes y una mortalidad de 2,5 a Io \%, y la hemorragia baja, una incidencia anual de 20 a 27 por Ioo.00o y una mortalidad de 4 a IO $\%{ }^{2-4}$.

Las causas de la hemorragia digestiva alta son: la enfermedad ulceropéptica, los tumores malignos gastroduodenales, el síndrome de MalloryWeiss y las lesiones de Dieulafoy, entre otras. Las de la hemorragia baja incluyen la enfermedad diverticular, las angiodisplasias, el cáncer de colon y la enfermedad inflamatoria intestinal. Normalmente, la hemorragia digestiva baja es de resolución espontánea y se trata médicamente; no obstante, en Io a I $5 \%$ de los casos es masiva, genera inestabilidad hemodinámica y pone en riesgo la vida del paciente ${ }^{3,4}$.

A pesar del uso de medicamentos supresores de la secreción gástrica y los tratamientos endoscópicos avanzados, no se puede controlar la hemorragia en Io \% de los casos y su reincidencia es de 5 a $25 \%$, lo que aumenta la mortalidad. Los factores predictores de reincidencia de la hemorragia después de la endoscopia, son: inestabilidad hemodinámica, úlceras de más de dos $\mathrm{cm}$, úlceras de la curva mayor del estómago o del duodeno, hemoglobina menor de Io g/dl y necesidad de transfusión; en estos casos, se contempla el uso de la formación de émbolos o de una cirugía mayor.

La formación de émbolos de los vasos mesentéricos se viene practicando desde hace más de 20 años; no obstante, muchos cirujanos no disponen de esta opción o no la conocen; por lo tanto, es importante usarla y estimular su realización en los casos indicados. Es una buena opción terapéutica antes del manejo quirúrgico, el cual tiene una mortalidad de más del $30 \%$ y solo se debe considerar como último recurso ${ }^{3-5}$.

El objetivo de este estudio fue determinar la indicación y el éxito del tratamiento endovascular para la hemorragia digestiva en el Hospital Universitario Clínica San Rafael.

\section{Materiales y métodos}

Se realizó un estudio retrospectivo y descriptivo, de noviembre del 2015 a junio del 20I6. Se evaluaron la utilización y la efectividad del tratamiento endovascular de la hemorragia gastrointestinal persistente a pesar del manejo endoscópico. Se incluyeron Io pacientes ( 5 hombres y 5 mujeres) con hemorragia digestiva de diferentes etiologías.

A todos los pacientes se les practicó una endoscopia digestiva urgente y se trataron las lesiones por esta vía siempre que fue posible. Los pacientes presentaron reincidencia de la hemorragia, necesidad de más de tres unidades de glóbulos rojos empaquetados, hipotensión o imposibilidad de control de hemorragia por vía endoscópica, por lo cual se indicó el tratamiento endovascular antes de considerar una cirugía.

Se evaluaron las siguientes variables: causa de la hemorragia, comorbilidades asociadas, arteria comprometida, número de unidades de sangre transfundidas, hemoglobina antes y después de la formación de los émbolos, hallazgos y tratamiento endoscópicos, tiempo del procedimiento, necesidad de intervención quirúrgica o nueva formación de émbolos, éxito técnico del procedimiento, complicaciones, material utilizado para la formación de émbolos y mortalidad a los 30 días.

Todos los pacientes tuvieron un seguimiento mínimo de 30 días. Se clasificó el estudio como una investigación de riesgo leve y se obtuvo la autorización de acuerdo con los protocolos de Helsinki y la Ley 23 de I98I sobre el código de ética médica. El procedimiento establecido en este estudio solo se utilizó para el mismo y, en ningún momento, se compartió o empleó en otros proyectos. Se garantizó la confidencialidad de la información, y prevaleció el criterio del respeto de la dignidad, y la protección de los derechos y el bienestar de los pacientes.

La formación de émbolos se definió como la liberación intravascular de un agente sólido o líquido, con el objetivo de generar la oclusión vascular. Se consideró un éxito técnico la oclusión completa del vaso escogido, confirmada por arteriografía, y un éxito clínico, el cese de la hemorragia en un periodo no mayor de 30 días. La recaída se diagnosticó por la reaparición de la hemorragia, la disminución de la hemoglobina y la inestabilidad hemodinámica.

Los criterios de inclusión fueron: hemorragia gastrointestinal, endoscopia fallida para contro- 
lar la hemorragia o reincidencia en menos de 24 horas, inestabilidad hemodinámica (tensión arterial sistólica menor de $100 \mathrm{~mm} \mathrm{Hg}$, frecuencia cardiaca mayor de Ioo por minuto), y necesidad de más de tres unidades de glóbulos rojos empaquetados durante la reanimación.

Los criterios de exclusión fueron: choque (es indicación de cirugía inmediata), hemorragia por várices esofágicas y alguna contraindicación para el procedimiento endovascular.

\section{Técnica del procedimiento}

A través de la arteria femoral común, bajo anestesia local y con un introductor 5 French (Cordis-Johnson \& Johnson Medical), se accedió a la aorta abdominal con una guía hidrofílica de $0,035 \times 260 \mathrm{~cm}^{2}$ Cordis Aquatrak ${ }^{\mathrm{TM}}$ (Johnson \& Johnson Medical). Se obtuvo primero un aortograma abdominal con un catéter pigtail (Cordis Aquatrak $^{\mathrm{TM}}$ ) para evaluar la aorta abdominal suprarrenal e infrarrenal y localizar los vasos. Después, se canalizaron los troncos mesentéricos con un catéter cobra o Simmons $\mathrm{I}^{\mathrm{TM}}$ (Johnson \& Johnson Medical), según la arteria sospechosa de sangrar. Se procedió a practicar la angiografía con sustracción digital, con varias inyecciones y con fase venosa tardía. Una vez visualizada la hemorragia e identificada la arteria comprometida, se inyectó el agente escogido para generar la embolia, por medio de microcatéteres y microguías.

En este estudio, se utilizaron al menos dos agentes para generar la embolia (Coils-espirales, micropartículas, Gelfoam ${ }^{\mathrm{TM}}$ ), pues la utilización de uno solo aumenta el riesgo de reincidencia de la hemorragia y de falla del procedimiento. Se utilizaron técnicas de formación de microémbolos con microcatéteres Direction ${ }^{\mathrm{TM}}$ (Boston Scientific, Massachusetts), microguías, microespirales (microcoils) de platino y micropartículas Contour $^{\mathrm{TM}}$ (Boston Scientific) que permiten acceder a vasos de I a $2 \mathrm{~mm}$ de diámetro. Se usaron solo agentes sólidos para generar embolia.

\section{Resultados}

Se estudiaron diez pacientes, cinco hombres y cinco mujeres, cuya edad promedio fue de 59,8 años, con un rango de 40 a 82 años. La hemorragia gastrointestinal fue alta en ocho casos y baja en dos. Las causas de la hemorragia digestiva alta fueron enfermedad acidopéptica en seis pacientes y cáncer gástrico en dos, y la enfermedad diverticular causó hemorragia baja en dos. En todos los pacientes, las manifestaciones clínicas de la hemorragia incluyeron hipotensión arterial sistólica (menos de $100 \mathrm{~mm} \mathrm{Hg}$ ), taquicardia (frecuencia cardiaca mayor de Ioo por minuto) y palidez mucocutánea. Al ingreso, se requirieron de dos a seis unidades de glóbulos rojos, con un promedio de 3,4 .

La mayoría de los pacientes presentaban comorbilidades importantes, como hipertensión arterial sistémica $(n=6)$, cáncer gastrointestinal $(n=5)$, enfermedad coronaria $(n=3)$, diabetes $(n=3)$ e insuficiencia renal crónica en hemodiálisis $(n=I)$, y enfermedad diverticular $(n=2)$.

El hallazgo endoscópico en seis pacientes con hemorragia digestiva por enfermedad acidopéptica, fue la hemorragia activa proveniente de un vaso visible (IA en la clasificación de Forrest). Los pacientes con cáncer gastrointestinal presentaban hemorragia en capa y coágulos múltiples, y aquellos con enfermedad diverticular, sangre fresca en el colon derecho y el sigmoide, pero sin un foco claro. El procedimiento endoscópico de los pacientes con cáncer y enfermedad acidopéptica, incluyó inyección de adrenalina y láser de argón; a los casos de hemorragia digestiva baja, no se les intervino con ningún tratamiento (tabla I).

Se observó extravasación del medio de contraste durante la arteriografía en ocho de los casos; en dos no se evidenció la hemorragia, pero se ocluyó la arteria que parecía ser la responsable de la hemorragia durante la endoscopia.

La arteria más frecuentemente comprometida fue la gastroduodenal (figura I) en cinco casos. La rama ascendente de la arteria cólica derecha, la rama ascendente de la arteria cólica izquierda y la arteria esplénica, estuvieron comprometidas, cada una, en un caso (figura 2).

Se obtuvo éxito técnico, o sea, se pudo ocluir la arteria sangrante, en todos los casos. El tiempo 
Tabla 1. Causa de la hemorragia y hallazgo endoscópico

\begin{tabular}{llll}
\hline Causa de la hemorragia & Hallazgo endoscópico & Manejo & $\mathbf{n}$ \\
\hline Enfermedad acidopéptica & Forrest 1A & Adrenalina & 6 \\
Cáncer & Hemorragia en capa - coágulo & Láser de argón & 2 \\
Enfermedad diverticular del colon & Hemorragia reciente sin foco & Ninguno & 2 \\
\hline
\end{tabular}
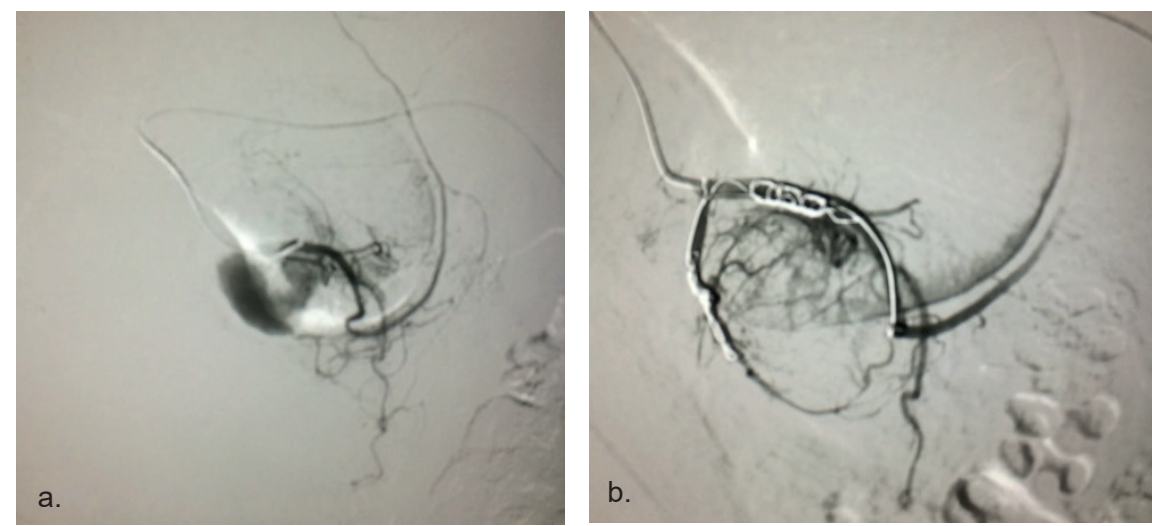

Figura 1. Hemorragia digestiva alta. a) Hemorragia proveniente de la arteria gastroduodenal. b) Control después de la formación de émbolos con microespirales y micropartículas.
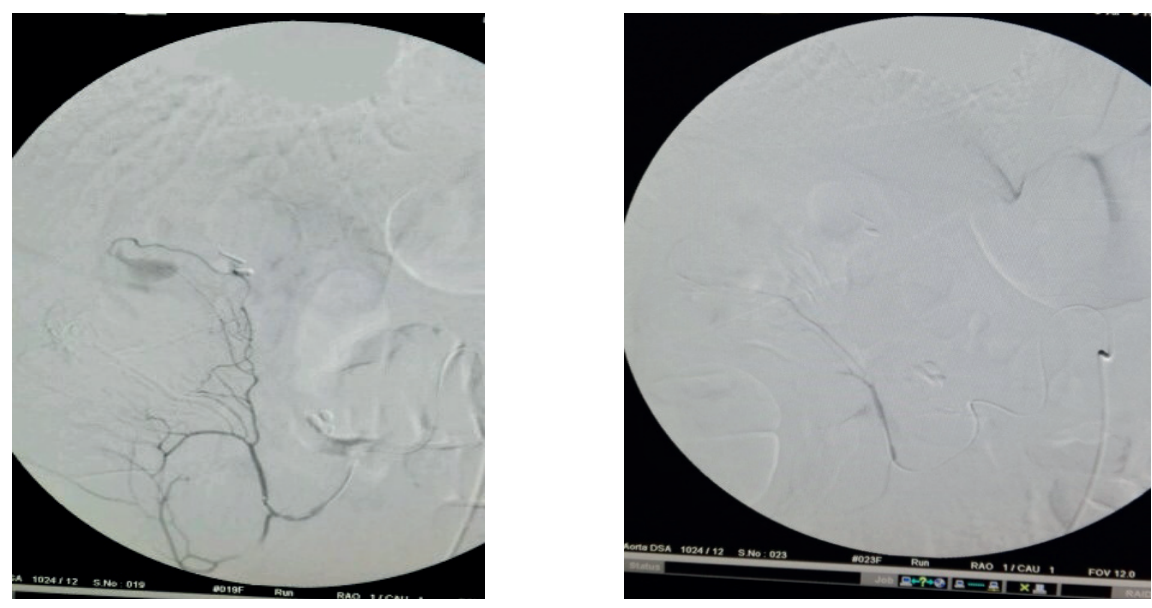

Figura 2. Hemorragia digestiva baja. a) Hemorragia proveniente del ángulo hepático del colon derecho. b) Control de la hemorragia después de la formación de émbolos.

promedio del procedimiento fue de 80 minutos, con un rango de 60 a 90 minutos. Se utilizaron diferentes agentes para causar la embolia, al menos, dos, en cada caso: Gelfoam ${ }^{\mathrm{TM}}$ en tres, espirales (coils) de 2 a $5 \mathrm{~mm}$ de diámetro en siete y micropartículas de 150 a $710 \mu \mathrm{m}$ en nueve.
No se presentaron complicaciones asociadas al procedimiento ni relacionadas con el acceso vascular, como hematomas, fístulas o hemorragias; tampoco, ninguna complicación como necrosis tisular o formación de émbolos de otro vaso diferente al objetivo. 
En todos los pacientes la concentración de hemoglobina aumentó y no se encontró descenso después de 24 a 48 horas del procedimiento; tampoco se presentaron nuevos episodios de hemorragia manifestados por hematemesis o rectorragia, lo cual corresponde a éxito clínico en todos los casos (figura 3). En ninguno de los Io casos, hubo necesidad de una intervención quirúrgica abierta para controlar la hemorragia.

Se presentaron tres muertes, pero estas no estuvieron asociadas a la hemorragia digestiva ni al procedimiento; se relacionaron con las comorbilidades de base de los pacientes: dos sufrieron infarto agudo de miocardio y uno falleció por falla multiorgánica secundaria a una infección intrabdominal. En la tabla 2 se presenta el resumen de los resultados por número de casos.

\section{Discusión}

La hemorragia gastrointestinal es desafiante para los médicos tratantes; es de suma importancia establecer un diagnóstico adecuado y rápido, para evitar complicaciones que pueden llevar a la muerte. La comunicación entre los especialistas y el uso de tecnologías avanzadas mínimamente invasivas, como la formación de émbolos, son muy útiles en el tratamiento de los pacientes y, además, evitan cirugías mayores que pueden tener complicaciones, aumento de los costos y una mortalidad alta.

El diagnóstico y el tratamiento dependen de la localización de la hemorragia, de su causa y de

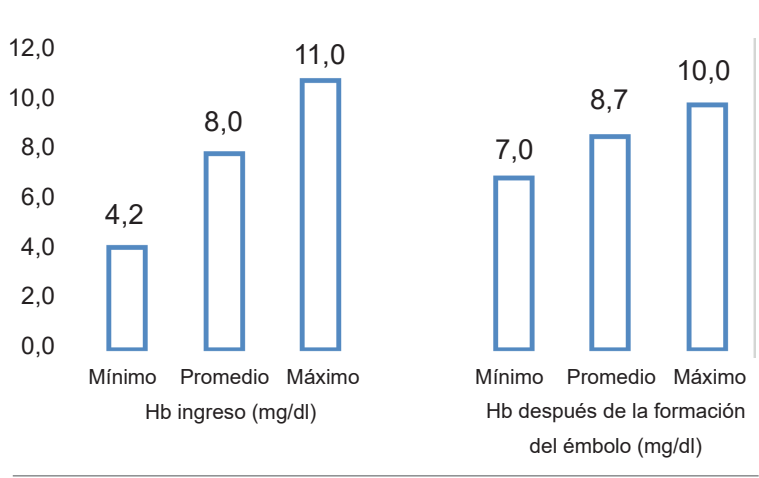

Figura 3. Concentración de hemoglobina antes y después del procedimiento (24-48 horas)
Tabla 2. Resultados principales

\begin{tabular}{|c|c|c|}
\hline \multirow{4}{*}{$\begin{array}{l}\text { Variable } \\
\text { Edad (años) }\end{array}$} & \multicolumn{2}{|l|}{ Valor y clasificación } \\
\hline & Promedio & 59,8 \\
\hline & Mínimo & 40 \\
\hline & Máximo & 82 \\
\hline \multirow[t]{5}{*}{ Comorbilidad } & Hipertensión arterial & 6 \\
\hline & Cáncer gastrointestinal & 5 \\
\hline & Enfermedad coronaria & 3 \\
\hline & Diabetes & 3 \\
\hline & Falla renal & 1 \\
\hline \multirow[t]{5}{*}{ Arteria comprometida } & Gastroduodenal & 5 \\
\hline & Esplénica & 1 \\
\hline & Rama cólica ascendente & 1 \\
\hline & Rama derecha cólica media & 1 \\
\hline & No se evidencia & 2 \\
\hline \multirow[t]{3}{*}{ Causa de la hemorragia } & Enfermedad acidopéptica & 6 \\
\hline & Cáncer & 2 \\
\hline & $\begin{array}{l}\text { Enfermedad diverticular del } \\
\text { colon }\end{array}$ & 2 \\
\hline \multirow[t]{3}{*}{ Unidades de transfusión } & Mínimo & 2,0 \\
\hline & Promedio & 3,4 \\
\hline & Máximo & 6,0 \\
\hline \multirow[t]{3}{*}{ Hemoglobina inicial (mg/dl) } & Mínimo & 4,2 \\
\hline & Promedio & 8,0 \\
\hline & Máximo & 11,0 \\
\hline \multirow{3}{*}{$\begin{array}{l}\text { Hemoglobina después del } \\
\text { procedimiento (mg/dl) }\end{array}$} & Mínimo & 7,0 \\
\hline & Promedio & 8,7 \\
\hline & Máximo & 10,0 \\
\hline \multirow{3}{*}{$\begin{array}{l}\text { Tiempo del procedimiento } \\
\text { (minutos) }\end{array}$} & Mínimo & 60 \\
\hline & Promedio & 80 \\
\hline & Máximo & 90 \\
\hline \multirow[t]{2}{*}{ Reintervención } & Sí & 0 \\
\hline & No & 10 \\
\hline \multirow[t]{2}{*}{ Complicaciones } & Sí & 0 \\
\hline & No & 10 \\
\hline \multirow[t]{2}{*}{ Mortalidad a 30 días } & Sí & 3 \\
\hline & No & 7 \\
\hline \multirow[t]{2}{*}{ Éxito técnico } & Sí & 10 \\
\hline & No & 0 \\
\hline \multirow[t]{2}{*}{ Necesidad de cirugía } & Sí & 0 \\
\hline & No & 10 \\
\hline \multirow{2}{*}{$\begin{array}{l}\text { Evidencia clínica de } \\
\text { control de la hemorragia }\end{array}$} & Sí & 0 \\
\hline & No & 10 \\
\hline \multirow[t]{3}{*}{ Material utilizado } & Gelfoam $^{\mathrm{TM}}$ & 3 \\
\hline & Coils o espirales & 7 \\
\hline & Micropartículas & 9 \\
\hline
\end{tabular}


su gravedad. Los procedimientos endovasculares han adquirido gran importancia, especialmente, cuando los métodos endoscópicos fallan. El acceso endovascular permite un tratamiento rápido, efectivo, mínimamente invasivo y repetible.

Los pacientes suelen consultar por manifestaciones clínicas como hematemesis, hematoquecia, melenas, hipotensión, taquicardia y anemia crónica. El manejo general de la hemorragia digestiva incluye reanimación hídrica, transfusión sanguínea y administración de inhibidores de la bomba de protones ${ }^{3-5}$. La endoscopia es el método de elección para el diagnóstico y el tratamiento de la hemorragia digestiva, especialmente para la alta; para la hemorragia baja, la colonoscopia es indispensable. La endoscopia tiene sensibilidad del $92 \%$ y especificidad del Ioo \% para identificar la causa de la hemorragia alta; la colonoscopia tiene una sensibilidad del $90 \%$ y un valor predictivo positivo del $87 \%$ para identificar la causa en casos de hemorragia baja ${ }^{5,6}$.

Las opciones terapéuticas endoscópicas que se tienen son: inyección de adrenalina, espirales o clips hemostáticos, electrocoagulación (gas de argón ionizado) y bandas.

Estos métodos son más fáciles y efectivos en la hemorragia digestiva alta que en la baja; es indispensable utilizar, al menos, dos procedimientos de control endoscópico del sangrado, pues uno solo generalmente es fallido ${ }^{7-9}$.

\section{Indicaciones de arteriografía y otros estudios para la hemorragia digestiva}

La arteriografía mesentérica y gastroduodenal para la ubicación y el tratamiento de la hemorragia digestiva, es una herramienta útil que fue descrita por primera vez por Rosch, et al., en I972. Se trata de un procedimiento rápido y con pocas complicaciones que, además de ser diagnóstico, es útil para el tratamiento, se puede repetir fácilmente y tiene pocas contraindicaciones 9 ,ro . Permite detectar una hemorragia activa con un flujo desde 0,5 a I $\mathrm{ml} /$ minuto.

La arteriografía mesentérica y la formación de émbolos están indicadas en pacientes con: alto riesgo de reincidencia de la hemorragia (clasifi- cación de Forrest: Ia, Ib; IIa y IIb); hemorragia masiva (transfusión mayor de tres unidades de glóbulos rojos en menos de 24 horas); hipotensión (tensión arterial sistólica $<\mathrm{IOO} \mathrm{mm} \mathrm{Hg}$, frecuencia cardiaca $>\mathrm{IOO} /$ por minuto), o hemorragia gastrointestinal resistente al tratamiento endoscópico o con gran riesgo de complicaciones y mortalidad por una intervención quirúrgica ${ }^{9-12}$.

La arteriografía se considera positiva cuando se observa extravasación del medio de contraste (6I \%) u otros signos indirectos, como aneurismas y pseudoaneurismas de las arterias mesentéricas, irregularidades de los vasos (estenosis y microaneurismas de las arterias viscerales), cortocircuitos arterio-venosos o arterio-portales, aumento de la irrigación por arteriolas dilatadas (típico de la angiodisplasia), llenado venoso precoz o aumento localizado de vasos sanguíneos.

Cuando se observa hemorragia activa en la endoscopia, pero no se demuestra durante la arteriografía, se recomienda la formación de émbolos "a ciegas", que consiste en ocluir la arteria correspondiente a la zona anatómica comprometida. En estos casos, se recomienda colocar un clip metálico donde el gastroenterólogo observe endoscópicamente la hemorragia, para facilitar la ubicación de la arteria sangrante.

\section{Complicaciones de la formación de émbolos}

Se presentan en el $9 \%$ de los casos, según la literatura científica. Además de las complicaciones propias de un acceso vascular, como hemorragia, hematoma, fístula arteriovenosa o disfunción renal, en este procedimiento puede presentarse la oclusión de una arteria diferente a la comprometida, disección arterial o necrosis intestinal del segmento tratado, infarto esplénico e infarto hepático; esto último sucede, especialmente, en pacientes con cirugía abdominal previa o radioterapia abdominal, en quienes la circulación colateral es deficiente, o cuando la generación de la embolia no es selectiva.

En el presente grupo de pacientes, no se presentaron complicaciones asociadas al acceso vascular; se procuró usar introductores de bajo perfil, de tipo 5 French. Además, se hicieron cana- 
lizaciones selectivas que permitieran acceder al vaso sangrante sin sacrificar la circulación colateral, para no generar necrosis intestinal, especialmente, en el colon donde el objetivo era ocluir los vasa recta. El síndrome posterior a la generación de la embolia se produce por la presencia de un cuerpo extraño dentro de la circulación arterial, y se manifiesta por un cuadro clínico de dolor abdominal, febrículas y leucocitosis, el cual se resuelve espontáneamente en dos a tres días ${ }^{10-12}$.

\section{Angiografía para estimular la hemorragia}

Es un procedimiento que se utiliza, como su nombre lo indica, para estimular la hemorragia cuando no se ve en la angiografía pues, en algunos casos, el sangrado es intermitente y no se evidencia al inyectar el medio de contraste. Se utilizan varios medicamentos (heparina, vasodilatadores, trombolíticos) en diferentes esquemas. Es una técnica poco usada y tiene el riesgo de empeorar el cuadro clínico al generar más hemorragia, aunque existen grupos que la practican, y sostienen que es segura y efectiva ${ }^{13}$.

\section{Técnicas de formación de émbolos}

El objetivo de la formación de émbolos es ocluir el vaso sangrante, sin generar necrosis tisular.

Existen tres técnicas de oclusión vascular en caso de hemorragia digestiva: la localizada, la proximal y la segmentaria. En la primera, la localizada, se ocluye de manera supraselectiva el vaso sangrante, sin sacrificar ninguna rama; el riesgo de necrosis o de reincidencia de la hemorragia es muy bajo. En la segunda, se ocluye la arteria proximal al sitio de la hemorragia y existe riesgo de recanalización. La tercera técnica es la segmentaria, en la cual se ocluyen la arteria que genera el sangrado y algunas otras adyacentes; en este caso, existe gran riesgo de recanalización ${ }^{\mathrm{I}, 15}$.

\section{Agentes para generar embolia}

Entre los múltiples agentes utilizados para ocluir los vasos sanguíneos, están las micropartículas, los espirales, los líquidos y el Gelfoam ${ }^{\mathrm{TM}}$ 15,16.
Las micropartículas de alcohol polivinílico tienen un diámetro que oscila entre las 200 y las $900 \mu \mathrm{m}$, son fáciles de usar, generan un control óptimo de la hemorragia, pero pueden aglutinarse en el catéter y ocluirlo, o producir reflujo al momento de la inyección y ocluir otros vasos. Las microesferas tienen un diámetro mejor calibrado, no se aglutinan tan fácilmente y son más fáciles de liberar, pero más costosas.

Los espirales o coils de acero inoxidable o platino están disponibles en diferentes diámetros y longitudes; permiten la oclusión mecánica, pero parcial, del vaso.

Los agentes líquidos generan un tapón completo del vaso, con riesgo de complicaciones isquémicas y estenosis crónicas; incluyen agentes como el Onyx ${ }^{\mathrm{TM}}$, el N-butil-cianoacrilato y el Glubran $^{\mathrm{TM}}$. Estos líquidos son costosos, de difícil control y su uso requiere un entrenamiento especial. El Glubran ${ }^{\mathrm{TM}}$ se ha asociado con estenosis duodenal meses después de su uso.

El Gelfoam ${ }^{\mathrm{TM}}$ es un agente derivado de la piel del cerdo y se usa como hemostático quirúrgico. No obstante, se puede utilizar para procedimientos endovasculares y tiene una gran incidencia de recanalización a las cuatro semanas; es fácil de usar y muy económico.

En este estudio, se utilizaron simultáneamente, al menos, dos de estos elementos para la oclusión vascular, ya que al usar uno solo se aumenta el riesgo de reincidencia de la hemorragia y de falla del procedimiento.

Cuando exista una circulación múltiple por ramas del tronco celiaco o de la arteria mesentérica superior o entre las arterias mesentéricas, se deben interrumpir ambos flujos, mediante lo que se conoce como la técnica del sándwich o back door. El caso típico es la oclusión de la arteria gastroduodenal para tratar úlceras duodenales o una enfermedad de la cabeza de páncreas; al mismo tiempo, se debe ocluir la arteria pancreático-duodenal superior, rama de la arteria mesentérica superior. $\mathrm{Si}$ no se interrumpe el flujo de estas dos arterias, el riesgo de reincidencia de la hemorragia es mayor.

Inicialmente, se usaron agentes vasoconstrictores, como la vasopresina, para controlar la 
hemorragia gastrointestinal. Sin embargo, esto generaba gran incidencia de complicaciones sistémicas (hipotensión, infarto agudo de miocardio, bradicardia), necrosis intestinal o gástrica, o reincidencia de la hemorragia, en 30 a $50 \%$ de los casos después de suspender el medicamento, por lo cual ya no se recomiendan ${ }^{17,18}$.

En varios estudios representativos sobre el tratamiento de la hemorragia gastrointestinal, que incluían 299 pacientes, se demostró: tasa de éxito técnico de 92 a Ioo \%, tasa de éxito clínico entre $5 \mathrm{I}$ y $94 \%$, reincidencia de la hemorragia entre 9 y $47 \%$, y mortalidad a 30 días de 3 a 27 $\%{ }^{17-19}$. En el presente estudio, el éxito clínico y la efectividad fueron del Ioo \%, al controlarse la hemorragia y no haber reincidencia.

Existen varios estudios retrospectivos en los cuales se compararon la cirugía y la oclusión vascular como tratamiento para la hemorragia digestiva alta. Se encontró que la oclusión vascular permite una mayor tasa de reincidencia de la hemorragia ( $28 \% V s$. $15 \%$ ) y la mortalidad fue igual con ambos procedimientos. No obstante, los pacientes sometidos a oclusión vascular son adultos mayores y con más comorbilidades; además, este procedimiento puede repetirse cuantas veces sea necesario, lo cual depende del éxito de la técnica y el material utilizados. Estos estudios están basados, únicamente, en técnicas con Gelfoam $^{\mathrm{TM}}$, el cual no es el mejor material para generar embolias y permite una gran incidencia de recanalización, y con técnicas no supraselectivas, lo que hace que falle más el procedimiento ${ }^{20,22}$. Hay estudios retrospectivos comparativos que sí muestran una incidencia mucho menor de complicaciones y control efectivo de la hemorragia con la formación de émbolos, en comparación con la cirugía abierta, como el de Wong, et al. $\mathrm{Al}$ comparar dos grupos de pacientes con hemorragia digestiva alta, uno de 26 tratados con oclusión vascular y otro de 56 con cirugía, estos autores encontraron complicaciones en el $40 \%$ del primer grupo y en el $70 \%$ del segundo ${ }^{20-22}$.

Por otra parte, la principal causa de hemorragia gastrointestinal baja, es la enfermedad diverticular, la cual se manifiesta con hemorra- gia profusa en 3 a Io \% de los pacientes; en estos casos, se trata de pseudodivertículos, porque no poseen todas las capas de tejido intestinal completas, de manera que el vaso no está protegido, se erosiona y sangra. La hemorragia es de resolución espontánea en el $90 \%$ de los casos $y$ es masiva en el Io \% ${ }^{23,24}$.

Los métodos endoscópicos, como la colonoscopia, detectan el foco exacto de la hemorragia en muy pocos casos (<40 \%), debido a la abundancia de materia fecal por falta de preparación del colon, por lo cual su papel en estos casos es solo diagnóstico y no terapéutico. Las indicaciones para la intervención endovascular en la hemorragia digestiva alta, son las mismas que en los casos de hemorragia masiva digestiva baja. Los métodos endovasculares en el tratamiento de la hemorragia digestiva baja también son una herramienta útil, ya que la colectomía en estos pacientes se acompaña de una mortalidad de más del $30 \%$ y de una morbilidad del $50 \%$. En diversos estudios se ha informado control de la hemorragia en 80 a IOo \% de los casos, riesgo de reincidencia en el $26 \%$, isquemia del colon en el I2 \%, y éxito técnico en el $97 \%{ }^{25-27}$.

Para el manejo endovascular de la hemorragia digestiva baja, las recomendaciones son utilizar microespirales (con menos de $4 \mathrm{~mm}$ de diámetro), y micropartículas entre 150 y $500 \mu \mathrm{m}$, y ocluir los vasos nutricios más cercanos al sitio de la hemorragia (vasa recta y arterias distales); los vasa recta son aquellos que dan origen a los vasos intramurales del intestino delgado o grueso. Además, se recomienda no colocar coils o espirales grandes en troncos primarios o secundarios, pues esto genera mayor riesgo de necrosis, aumento de la circulación colateral y hemorragia, e impide un nuevo acceso al sitio de la hemorragia ${ }^{27-32}$.

En conclusión, los procedimientos endovasculares son una herramienta útil en el diagnóstico y el tratamiento de la hemorragia gastrointestinal, disminuyen las complicaciones y la mortalidad, pueden repetirse en caso de una nueva hemorragia y deben llevarse a cabo en instituciones con experiencia, donde se cuente con profesionales entrenados en estas técnicas, y con los recursos 
tecnológicos y los materiales adecuados. Además, la comunicación es de vital importancia y la atención debe ser rápida para optimizar los resultados.

Se requieren más estudios aleatorios y a largo plazo para determinar si la embolización es mejor que la cirugía para controlar la hemorragia, aunque los estudios apuntan cada vez más a que la oclusión vascular tiene buena efectividad y seguridad, mayor que la de la cirugía y con menos complicaciones.

Conflicto de interés: Ninguno reportado por los autores.

Fuentes de financiación: Autofinanciado.

\section{Referencias}

I. Rivera D, Martínez J, Tovar J, Garzón M, Hormaza N, Lizarazo L, et al. Caracterización de los pacientes con hemorragia de vías digestivas altas no varicosa en un hospital de tercer nivel de Cundinamarca, Colombia. Rev Colomb Gastroenterol. 2013;28:278-85.

2. Manning-Dimmitt LL, Dimmitt SG, Wilson GR. Diagnosis of gastrointestinal bleeding in adults. Am Fam Physician. 2005;71:1339-46.

3. Longstreth GF. Epidemiology and outcome of patients hospitalized with acute lower gastrointestinal hemorrhage: A population-based study. Am J Gastroenterol. 1997;92:419-24.

4. Evangelista PT, Hallisey MJ. Transcatheter embolization for acute lower gastrointestinal hemorrhage. J Vasc Interv Radiol. 2000;II:60I-6.

5. Ahmed A, Stanley AJ. Acute upper gastrointestinal bleeding in the elderly: Aetiology, diagnosis and treatment. Drugs Aging. 2012;29:933-40.

6. Srygley FD, Gerardo CJ, Tran T, Fisher DA. Does this patient have a severe upper gastrointestinal bleed? JAMA. 2012;307:1072-9.

7. Wilkins T, Khan N, Nabh A, Schade RR. Diagnosis and management of upper gastrointestinal bleeding. Am Fam Physician. 20I2;85:469-76.

8. Smith GA, O'Dwyer PJ. Sensitivity of double contrast barium enema and colonoscopy for the detection of colorectal neoplasms. Surg Endosc. 200I;15:649-65.

9. Zuckerman GR, Prakash C, Askin MP, Lewis BS. AGA technical review on the evaluation and management of occult and obscure gastrointestinal bleeding. Gastroenterology. 2000;II8:20I-2I.

Io. Zuccaro G. Management of the adult patient with acute lower gastrointestinal bleeding. American College of Gastroenterology. Practice Parameters Committee. Am J Gastroenterol. 1998;93:I202-8.
II. Shin JH. Recent update of embolization of upper gastrointestinal tract bleeding. Korean J Radiol. 20I2;I3:S3I-9. doi: I0.3348/kjr.20I2.I3.SI.S3I.

12. Walsh RM, Anain P, Geisinger M, Vogt D, Mayes J, Grundfest- Broniatowski S, et al. Role of angiography and embolization for massive gastroduodenal hemorrhage. J Gastrointest Surg. 1999.3:6I-6.

13. Lu Y, Loffroy R, Lau JY, Barkun A. Multidisciplinary management strategies for acute non-variceal upper gastrointestinal bleeding. Br J Surg. 20I4;IOI:e34-50.

I4. Beltrán R, Cordero R, Maldonado P, Marcos F, Romero $\mathrm{S}$, Pellicer F, et al. Embolización (sic) en pacientes con hemorragia digestiva no varicosa refractaria (sic) al manejo endoscópico. Revista Andaluza de Patología Digestiva. 2012;35:450-4. Consultado: $\mathrm{I}^{\circ}$ de enero de 20I9. Disponible en: https://dialnet.unirioja.es/servlet/ articulo? codigo $=4099340$

I5. Ryan JM, Key SM, Dumbleton SA, Smith TP. Nonlocalized lower gastrointestinal bleeding: Provocative bleeding studies with intraarterial tPA, heparin, and tolazoline. J Vasc Interv Radiol. 200I;I2:1273-7.

I6. Song JS, Kwak HS, Chung GH. Nonvariceal upper gastrointestinal bleeding: The usefulness of rotational angiography after endoscopic marking with a metallic clip. Korean J Radiol. 20II;12:473-80.

17. Gomes AS, Lois JF, McCoy RD. Angiographic treatment of gastrointestinal hemorrhage: Comparison of vasopressin infusion and embolization. Am J Roentgenol. I986;I46:IO3I-7. doi: I0.22I4/ajr.I46.5.IO3I.

I8. Gwon DI, Ko GY, Sung KB, Shin JH, Kim JH, Yoon HK. Endovascular management of extrahepatic artery hemorrhage after pancreatobiliary surgery: Clinical features and outcomes of transcatheter arterial embolization and stent-graft placement. Am J Roentgenol. 20II;196:W627-34.

19. Loffroy R, Guiu B, D'Athis P, Mezzetta L, Gagnaire A, Jouve JL, et al. Arterial embolotherapy for endoscopically unmanageable acute gastroduodenal hemorrhage: Predictors of early rebleeding. Clin Gastroenterol Hepatol. 2009;7:515-23.

20. Ramaswamy RS, Won HC, Mouser HC, Narsinh KH, McCammack KC, Treesit T, et al. Role of interventional radiology in the management of acute gastrointestinal bleeding. World J Radiol. 20I4;6:82-92. doi: I0.4329/wjr.v6.i4.82.

2I. Holme JB, Nielsen DT, Funch-Jensen P, Mortensen FV. Transcatheter arterial embolization in patients with bleeding duodenal ulcer: An alternative to surgery. Acta Radiol. 2006;47:244-7.

22. Wong TC, Wong KT, Chiu PW, Teoh AY, Yu SC, Au KW, et al. A comparison of angiographic embolization with surgery after failed endoscopic hemostasis to bleeding peptic ulcers. Gastrointest Endosc. 20II;73:900-8.

23. Poultsides GA, Kim CJ, Orlando R, Peros G, Hallisey MJ, Vignati PV. Angiographic embolization for gastroduodenal hemorrhage: Safety, efficacy, and predictors of outcome. Arch Surg. 2008;I43:457-6I. 
24. Larssen L, Moger T, Bjornbeth BA, Lygren I, Kløw NE. Transcatheter arterial embolization in the management of bleeding duodenal ulcers: A 5.5-year retrospective study of treatment and outcome. Scand J Gastroenterol. 2008;43:217-22.

25. Ang D, Teo EK, Tan A, Ibrahim S, Tan PS, Ang TL, et al. A comparison of surgery versus transcatheter angiographic embolization in the treatment of nonvariceal upper gastrointestinal bleeding uncontrolled by endoscopy. Eur J Gastroenterol Hepatol. 2012;24:929-38.

26. Venclauskas L, Bratlie SO, Zachrisson K, Maleckas A, Pundzius J, Jonson C. Is transcatheter arterial embolization a safer alternative than surgery when endoscopic therapy fails in bleeding duodenal ulcer? Scand J Gastroenterol. 2010;45:299-304.

27. Ripoll C, Banares R, Beceiro I, Menchen P, Catalina $\mathrm{MV}$, Echenagusia A, et al. Comparison of transcatheter arterial embolization and surgery for treatment of bleeding peptic ulcer after endoscopic treatment failure. J Vasc Interv Radiol. 20I4;15:447-50.
28. Eriksson LG, Ljungdahl M, Sundbom M, Nyman R. Transcatheter arterial embolization versus surgery in the treatment of upper gastrointestinal bleeding after therapeutic endoscopy failure. J Vasc Interv Radiol. 2008;I9:I4I3-8. doi: I0.IOI6/j.jvir.2008.06.0I9.

29. Maykel J, Opelka G. Colonic diverticulosis and diverticular hemorrhage. Clin Colon Rectal Surg. 2004;I7:I95204. doi: I0.IO55/s-2004-832702.

30. Bertrand J, Surapaneni B, Rabkin D, Nasser I, Clouse M. Microcoil embolization for acute lower gastrointestinal bleeding. Cardiovasc Intervent Radiol. 2006;29:49-58.

3I. Ker-Kan T, Wong D, Sim R. Superselective embolization for lower gastrointestinal hemorrhage: An institutional review over 7 years. World J Surg. 2008;32:2707-I5.

32. García-Mónaco R, Peralta O, Fernández-Antruejo L, Bonadeo F, Rossi G, San Román E, et al. Embolización superselectiva en el tratamiento de la hemorragia digestiva baja masiva. Acta Gastroenterol Latinoam. 2OII;4I:II9-27. 